\title{
Risk and Cumulative Risk of Acute Ischemic Stroke Recurrence in China:A Systematic Review and Meta-Analysis
}

\author{
You-Hua LIU ${ }^{1}$, Hong GUO ${ }^{1, *}$, Gui-Ying LIU², Luo YANG ${ }^{1}$, Hai-Yan WANG ${ }^{1}$, Dan ZHAO ${ }^{1}$, \\ Hui JU' ${ }^{1}$, Shao-Hua GONG ${ }^{1}$, Xiao-Ping $\mathrm{YI}^{1}$ \\ ${ }^{1}$ School of Nursing, Beijing University of Chinese Medicine, Beijing 102448, China; \\ ${ }^{2}$ Department of Brain Surgery, Oriental Hospital, Beijing University of Chinese Medicine, Beijing 100078, China
}

\begin{abstract}
Objective: To comprehensively evaluate the risk of recurrence after initial ischemic stroke, and to provide a relatively comprehensive reference for the prevention and control of stroke recurrence.

Methods: CNKI, VIP, Wanfang, PubMed, Web of Science, Embase and other databases were collected to investigate the status of recurrence after initial ischemic stroke, the search period of which was from the establishment of databases to March 2019. And then quality evaluation and data extraction were carried out. The overall cumulative risks of stroke recurrence at 3 months, 6 months, 1 year, 2 years and 5 years after initial ischemic stroke were calculated, and heterogeneity analysis was performed.

Results: A total of 29 studies from 19 provinces (cities, autonomous regions) in China were included. The cumulative total sample size was 22484 cases, the cumulative recurrent sample size was 3309 cases. The pooled cumulative risk was $4.5 \%$ (95\% CI: 3.1-5.8) at 3 months, $7.8 \%$ (95\% CI: 5.6-10.0) at 6 months, $13.6 \%$ (95\% CI: $11.0-16.2)$ at 1 year, $17.5 \%$ (95\% CI: $12.4-22.6)$ at 2 years and $30.9 \%$ (95\% CI: $20.2-41.7)$ at 5 years after initial ischemic stroke.

Conclusions: The recurrence rate of acute ischemic stroke in China is high. It is recommended that all levels of medical and health departments strengthen the prevention and treatment of ischemic stroke recurrence to reduce the recurrence of ischemic stroke and improve the prognosis of patients.
\end{abstract}

Key words: Acute ischemic stroke; Recurrence; Meta-analysis; Frequency

\section{Introduction}

Acute ischemic stroke (AIS) has long been the focus of global stroke prevention and control, which is attributed to its high disability, high mortality and high recurrence rate.

\section{Corresponding Author:}

Hong GUO,Associate professor, Clinical Nursing Department, School of Nursing, Beijing University of Chinese Medicine, Beijing 102448, China E-mail: guohong2015@I63.com

Received:April 8 2019, Revised:April 15 2019,Accepted:April 20 2019, Online: May 202019
The recurrence of AIS will aggravate the deterioration of neurological function, and increase the risk of bad clinical outcome of stroke. It brings irreparable loss to the patients' life and health. The overall situation of stroke recurrence has been reported in foreign countries ${ }^{[1]}$. Although a large number of epidemiological investigations have been carried out on the recurrence of AIS in China, 
there is still a lack of overall description and systematic evaluation of the related situation. Based on this, a metaanalysis was used to comprehensively analyze the pooled cumulative risk of stroke recurrence at 3,6 months and $1,5,10$ years after initial ischemic stroke in China, in order to fully understand the epidemic situation of stroke recurrence in China in an all-round way. It provides a relatively comprehensive reference for medical workers and researchers.

\section{Materials and Methods}

\section{Search strategy}

The literatures on the risk of recurrence of acute ischemic stroke were systematically collected according to the principle of free subject words. The sources of the literatures included CNKI, Wanfang Database, VIP Database, PubMed, Web of Science, The Cochrane Library, CINAHL, EMbase. And the search period was from the establishment of databases to March 2019. Chinese retrieval terms included: “中风/缺血性中风/ 脑血管病 / 脑血管意外/脑栓塞 / 脑血栓 / 脑梗塞”, “复 发”. English retrieval terms included: “stroke / ischemic stroke / ischemia cerebra / cerebral ischemia / cerebral infarction / cerebrovascular disease /cerebral vascular accident/ cerebrovascular accident /brain vascular accident / PostStroke", "recurrent / recurrence". In addition, there was in the way of snowball to trace back to the citation.

\section{Correlation definition}

Initial ischemic stroke: The patients with initial stroke were diagnosed according to the diagnostic criteria of ischemic stroke established by the $4^{\text {th }}$ National Conference on Cerebrovascular Diseases in 1995 and supported by CT or MRI.

Recurrent ischemic stroke: The initial attack was relieved, and more than 28 days later, the new central nervous system damage appeared again, the localization signs or the original symptoms were aggravated, which was confirmed by the skull CT or MRI.

\section{Selection Criteria}

Inclusion criteria: (1) Chinese stroke patients were diagnosed in China; (2) There were clear diagnoses of ischemic stroke and its recurrence; (3) The study was designed as a cohort study; (4) The recurrence rate of ischemic stroke was provided directly or indirectly.

Exclusion criteria: (1) Lectures, reviews, case reports, etc; (2) Experimental studies of basic medicine; (3) Literatures with repeated publication of the same data, incomplete original data, no full text, unclear types of research, and erroneous data statistics.

\section{Literature screening and data extraction}

Endnote X8 was used for screening and data extraction, and 2 researchers independently browsed the papers and abstracts for the preliminary screening, read through the full text and completed the re-screening. In case of disagreement, the third party decided. Data extraction included: first author, published year, region, recurrence cases, total follow-up cases, etc.

\section{Literature quality evaluation}

The Newcastle-Ottawa quality assessment table (Newcastle-Ottawa Scale, NOS) was used to evaluate the quality of the included literature based on the following 3 aspects: the selection of the study population, the comparability between groups, and the exposure factors. Those with score $\geq 5$ pointscould be included in meta analysis. If the score was more than 5 points, the meta analysis could be carried out. The 2 researchers independently and mutual-blindly completed the process, and then compared the evaluation results with each other. In case of disagreement, the results were decided by a third party.

\section{Statistical analyses}

The double-chord transform method was used to transform the data. And then Metaprop of the Stata software was used to calculate the progressive rate of the data. The heterogeneity between the included literature was tested by $\mathrm{I}^{2}$ value and Q test. When $P>0.1, \mathrm{I}^{2} \leq 50 \%$, the studies was considered to be homogeneous, and then the fixed effect model was used to combine the effect values, otherwise the random effect model was used. The sensitivity analysis was carried out to verify the stability 
of the combined effect values by item-by-item exclusion of the single included study. The publication bias was quantitatively evaluated based on the Egger test. The difference was statistically significant when $P<0.05$.

\section{Results}

\section{Literature inclusion and quality evaluation}

Three-thousand five-hundred eighty-one studies were identified by the electronic database searches. After rechecking, browsing the abstracts, reading the full text and other layers of screening, there was finally ended up with 29 documents $^{[3-31]}$. The cumulative total sample size was 22484 cases, and the cumulative recurrent sample size was 3142 cases. The recurrent time of the subjects ranged from 1991 to 2016, and the follow-up time ranged from 3 months to 5 years. The research sites covered 19 provinces in China. The quality evaluation score of the included literature was at least 5 points, with a maximum of 8 points. The estimates of risk of acute ischemic stroke recurrence across all the included studies was shown in Figure 1.

\section{Meta analysis results}

The recurrence rateat 3 months after first-ever AIS: 6 studies ${ }^{[6,14,20,21,25,31]}$ (a total of 4450 patients) reported the recurrence rate at 3 months after AIS, with a cumulative recurrence rate of $2.28 \%$ to $8.52 \%$. Statistical heterogeneity was found among the studies $(P=0.001$, $\mathrm{I}^{2}=76.1 \%$ ), so random effect model was used to analyze the combined effects. The results showed that the cumulative recurrence rate was $4.5 \%$ (95\% CI: 3.1-5.8) at 3 months afterinitialAIS, as shown in Figure 2.

The recurrence rate at 6 months after first-ever AIS: 7 studies $^{[6,14,18,20,21,25,31]}$ (a total of 4626 patients) reported the recurrence rateat 6 months after acute ischemic stroke, with a cumulative recurrence rate of $4.39 \%$ to $14.07 \%$. Statistical heterogeneity was found among the studies $\left(P<0.001, \mathrm{I}^{2}=86.1 \%\right)$, so random effect model was used to analyze the combined effects. The results showed that the cumulative recurrence rate was $7.8 \%$ (95\% CI: 5.6-10.0) at 6 months after first-ever AIS, as shown in Figure 3.

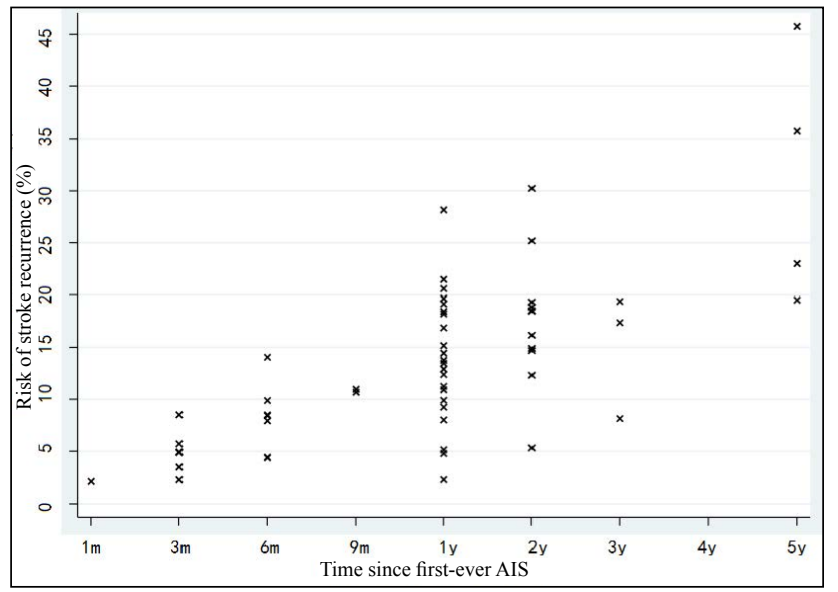

Figure 1 Risk of AIS recurrence across all studies

Notes: "m" refers to month(s); " $y$ " refers to year(s)

The recurrence rateat 1 year after first-ever AIS: 23 studies $^{[3,6,8,10-17,19-27,29-31]}$ (a total of 20618 patients) reported the recurrence rate of AIS at 1 year after acute ischemic stroke, with a cumulative recurrence rate of $4.8 \%$ to $28.19 \%$. Statistical heterogeneity was found among the studies ( $P=0.000, \mathrm{I}^{2}=97.9 \%$ ), so random effect model was used to analyze the combined effects. The results showed that the cumulative recurrence rate was $13.6 \%$ (95\% CI: 11.0-16.2) at 1 year after first-ever AIS, as shown in Figure 4.

The recurrence rate at 2 years after first-everAIS: 11 studies $^{[4-5,7,10-11,13,15,20,22,24,28]}$ (a total of 11427 patients) reported the recurrence rate at 2 years after initial AIS, with a cumulative recurrence rate of $5.34 \%$ to $30.26 \%$. Statistical heterogeneity was found among the studies $(P<0.001$, $\mathrm{I}^{2}=97.8 \%$ ), so random effect model was used to analyze the combined effects. The results showed that the cumulative recurrence rate was $17.5 \%$ (95\% CI: 12.4-22.6) at 2 years after initial AIS, as shown in Figure 5.

The recurrence rateat 5 years after first-everAIS: 4 studies ${ }^{[9,13,19-20]}$ (a total of 2508 patients) reported the recurrence rateat 5 years after initial AIS, with a cumulative recurrence rate of $19.55 \%$ to $45.78 \%$. Statistical heterogeneity was found among the studies $\left(P<0.001, \mathrm{I}^{2}=97.3 \%\right)$, so random effect model was used to analyze the combined effects. The results showed that the cumulative recurrence rate was $30.9 \%$ (95\% CI: $20.2-$ 41.7) at 5 years after AIS, as shown in Figure 6. 
Table 1 The basic situation and quality evaluation of AIS recurrence risk in literature

\begin{tabular}{|c|c|c|c|c|c|c|c|c|c|c|c|}
\hline \multirow{2}{*}{ Authors } & \multirow{2}{*}{$\begin{array}{c}\text { Year of } \\
\text { publication }\end{array}$} & \multirow{2}{*}{$\begin{array}{l}\text { Initial study } \\
\text { period }\end{array}$} & \multirow{2}{*}{ Area } & \multicolumn{2}{|c|}{ Sample size } & \multicolumn{5}{|c|}{$\begin{array}{c}\text { Cumulative risk of stroke } \\
\text { recurrence (\%) }\end{array}$} & \multirow{2}{*}{ NOS score } \\
\hline & & & & $\begin{array}{l}\text { Recurrent } \\
\text { cases }\end{array}$ & $\begin{array}{l}\text { Total } \\
\text { cases }\end{array}$ & $3 \mathrm{~m}$ & $6 \mathrm{~m}$ & $1 \mathrm{y}$ & $2 y$ & $5 y$ & \\
\hline Chen Peng ${ }^{[3]}$ & 2014 & 2011-2012 & Ningxia & 79 & 401 & $\ldots$ & $\ldots$ & 79 & $\ldots$ & $\ldots$ & 7 \\
\hline Zheng Jianghuan ${ }^{[4]}$ & 2015 & $2010-2013$ & Guizhou & 30 & 162 & $\ldots$ & $\ldots$ & $\ldots$ & 30 & $\ldots$ & 8 \\
\hline $\mathrm{Li} \mathrm{Qi}^{[5]}$ & 2014 & 2011-2014 & Hainan & 27 & 143 & $\ldots$ & $\ldots$ & $\ldots$ & 27 & $\ldots$ & 8 \\
\hline Luan $\mathrm{Mei}^{[6]}$ & 2016 & 2013-2015 & Chongqing & 41 & 270 & 23 & 38 & 41 & $\ldots$ & $\ldots$ & 8 \\
\hline Su Changqing ${ }^{[7]}$ & 2014 & 2011-2014 & Fujian & 31 & 123 & $\ldots$ & $\ldots$ & $\ldots$ & 31 & $\ldots$ & 7 \\
\hline Song $\mathrm{Li}^{[8]}$ & 2008 & $2005-2007$ & Liaoning & 58 & 421 & $\ldots$ & $\ldots$ & 58 & $\ldots$ & $\ldots$ & 8 \\
\hline Cheng Yuefeng ${ }^{[9]}$ & 2017 & 2011-2016 & Heilongjiang & 206 & 450 & $\cdots$ & $\ldots$ & $\ldots$ & $\ldots$ & 206 & 7 \\
\hline An Yachen ${ }^{[10]}$ & 2017 & 2013-2016 & Hebei & 184 & 1058 & $\ldots$ & $\ldots$ & 98 & 158 & $\ldots$ & 8 \\
\hline Chen Yunxia ${ }^{[11]}$ & 2011 & $2008-2010$ & Hebei & 79 & 408 & $\ldots$ & $\ldots$ & 55 & 79 & $\ldots$ & 8 \\
\hline Zhang Shenning ${ }^{[12]}$ & 2008 & 2004-2006 & Jiangsu & 160 & 834 & $\ldots$ & $\ldots$ & 160 & $\ldots$ & $\ldots$ & 7 \\
\hline Zheng Shengbang ${ }^{[13]}$ & 2017 & $2010-2015$ & Shanghai & 233 & 651 & $\ldots$ & $\ldots$ & 140 & 197 & 233 & 7 \\
\hline Wang Jing ${ }^{[14]}$ & 2016 & 2013-2015 & Zhejiang & 35 & 282 & 14 & 24 & 35 & $\ldots$ & $\ldots$ & 8 \\
\hline Tang Meilian ${ }^{[15]}$ & 2016 & 2014-2015 & China & 647 & 6450 & $\ldots$ & $\ldots$ & 148 & 345 & $\ldots$ & 8 \\
\hline Zhang Changqing ${ }^{[16]}$ & 2018 & 2007-2009 & China & 95 & 1978 & $\ldots$ & $\ldots$ & 95 & $\ldots$ & $\ldots$ & 7 \\
\hline Zhao Yan ${ }^{[17]}$ & 2014 & $2010-2012$ & Henan & 105 & 812 & $\ldots$ & $\ldots$ & 105 & $\ldots$ & $\ldots$ & 6 \\
\hline Ma Juanjuan ${ }^{[18]}$ & 2015 & $2013-2015$ & Jiangsu & 14 & 176 & $\ldots$ & 14 & $\ldots$ & $\ldots$ & $\ldots$ & 5 \\
\hline Yan Zhongrui ${ }^{[19]}$ & 1998 & 1991-1996 & Shandong & 112 & 573 & $\cdots$ & $\ldots$ & 46 & $\cdots$ & 112 & 7 \\
\hline Wang Liping ${ }^{[20]}$ & 2005 & 1994-1999 & Henan & 192 & 834 & 19 & 37 & 94 & 135 & 192 & 6 \\
\hline Duan Kangli ${ }^{[21]}$ & 2018 & 2014-2015 & Shanxi & 115 & 2230 & 78 & 98 & 115 & $\ldots$ & $\ldots$ & 6 \\
\hline Yang Junping ${ }^{[22]}$ & 2014 & $2009-2013$ & Henan & 97 & 786 & $\ldots$ & $\ldots$ & 78 & 97 & $\ldots$ & 5 \\
\hline Zhang Ming ${ }^{[23]}$ & 2016 & $2010-2015$ & Shandong & 49 & 269 & $\ldots$ & $\ldots$ & 49 & $\ldots$ & $\ldots$ & 6 \\
\hline Xue Min ${ }^{[24]}$ & 2013 & $2008-2011$ & Anhui & 90 & 612 & $\ldots$ & $\ldots$ & $\ldots$ & 90 & $\ldots$ & 6 \\
\hline Wang Xiaoqing ${ }^{[25]}$ & 2018 & 2014-2016 & Hebei & 76 & 450 & 22 & 38 & 76 & $\ldots$ & $\ldots$ & 6 \\
\hline Chen Jingfei ${ }^{[26]}$ & 2015 & $2012-2014$ & Ningxia & 43 & 233 & $\ldots$ & $\ldots$ & 43 & $\ldots$ & $\ldots$ & 5 \\
\hline Xu Lina ${ }^{[27]}$ & 2018 & 2014-2016 & Shanxi & 45 & 311 & $\ldots$ & $\ldots$ & 45 & $\ldots$ & $\ldots$ & 5 \\
\hline Zhang Youlin ${ }^{[28]}$ & 2012 & $2007-2009$ & Beijing & 37 & 200 & $\ldots$ & $\ldots$ & $\ldots$ & 37 & $\ldots$ & 6 \\
\hline Sun Shuju ${ }^{[29]}$ & 2013 & $2008-2011$ & Hubei & 42 & 149 & $\ldots$ & $\ldots$ & 42 & $\ldots$ & $\ldots$ & 5 \\
\hline Gelin $\mathrm{Xu}{ }^{[30]}$ & 2007 & $2003-2006$ & Jiangsu & 172 & 834 & $\ldots$ & $\ldots$ & 172 & $\ldots$ & $\ldots$ & 7 \\
\hline Wang Weiying ${ }^{[31]}$ & 2016 & $2014-2015$ & Beijing & 42 & 384 & 22 & 38 & 42 & $\ldots$ & $\ldots$ & 5 \\
\hline
\end{tabular}

Notes: "m" refers to month(s); "y" refers to year(s) 


\begin{tabular}{|c|c|c|c|}
\hline $\begin{array}{l}\text { Study } \\
\text { ID }\end{array}$ & & ES $(95 \% \mathrm{Cl})$ & $\begin{array}{l}\% \\
\text { Weight }\end{array}$ \\
\hline Iuan Mei 2016 & & $0.09(0.05,0.12)$ & 10.04 \\
\hline Wang Jing 2016 & & $0.05(0.02,0.07)$ & 13.45 \\
\hline Wang Liping 2005 & $\rightarrow$ & $0.02(0.01,0.03)$ & 22.18 \\
\hline Duan Kangli 2018 & $\rightarrow$ & $0.04(0.03,0.04)$ & 23.43 \\
\hline Wang Xiaoqing 2018 & & $0.05(0.03,0.07)$ & 16.38 \\
\hline Wang Weiying 2016 & & $0.06(0.03,0.08)$ & 14.53 \\
\hline Overall $(I-$ squared $=76.1 \%, p=0.001)$ & & $0.04(0.03,0.06)$ & 100.00 \\
\hline
\end{tabular}

Figure 2 Cumulative risk of stroke recurrence 3 months after first-ever AIS

\begin{tabular}{|c|c|c|c|}
\hline $\begin{array}{l}\text { Study } \\
\text { ID }\end{array}$ & & $\mathrm{ES}(95 \% \mathrm{Cl})$ & $\begin{array}{l}\% \\
\text { Weight }\end{array}$ \\
\hline Iuan Mei 2016 & & $0.14(0.10,0.18)$ & 11.19 \\
\hline Wang Jing 2016 & & $0.09(0.05,0.12)$ & 13.21 \\
\hline Wang Juanjuan 2016 & & $0.08(0.04,0.12)$ & 11.51 \\
\hline Wang Liping 2005 & $\rightarrow-$ & $0.04(0.03,0.06)$ & 17.31 \\
\hline Duan Kangli 2018 & + & $0.04(0.04,0.05)$ & 18.11 \\
\hline Wang Xiaoqing 2018 & & $0.08(0.06,0.11)$ & 14.84 \\
\hline Wang Weiying 2016 & & $0.10(0.07,0.13)$ & 13.84 \\
\hline Overall $(\mathrm{I}$-squared $=86.1 \%, p=0.000$ ) & & $0.08(0.06,0.10)$ & 100.00 \\
\hline
\end{tabular}

Figure 3 Cumulative risk of stroke recurrence 6 months after first-ever AIS

\begin{tabular}{|c|c|c|c|}
\hline \multicolumn{2}{|l|}{ Study } & \multicolumn{2}{|r|}{$\%$} \\
\hline ID & & ES $(95 \% \mathrm{Cl})$ & Weight \\
\hline Chen Peng 2014 & $\rightarrow$ & $0.20(0.16,0.24)$ & 4.42 \\
\hline Iuan Mei 2016 & $\rightarrow-$ & $0.15(0.11,0.19)$ & 4.33 \\
\hline Song Li 2008 & & $0.14(0.10,0.17)$ & 4.55 \\
\hline An Yachen 2017 & +1 & $0.09(0.08,0.11)$ & 4.80 \\
\hline Chen Yunxia 2011 & $\longrightarrow$ & $0.13(0.10,0.17)$ & 4.54 \\
\hline Zhang Shenning 2008 & $1-$ & $0.19(0.17,0.22)$ & 4.66 \\
\hline Zheng Shengbang 2017 & $i \multimap$ & $0.22(0.18,0.25)$ & 4.57 \\
\hline Wang Jing 2016 & + & $0.12(0.09,0.16)$ & 4.43 \\
\hline Tang Meilian 2016 & $\bullet$ & $0.02(0.02,0.03)$ & 4.90 \\
\hline Zhang Changqing 2018 & $\bullet$ & $0.05(0.04,0.06)$ & 4.87 \\
\hline Zhao Yan 2014 & $\rightarrow$ & $0.13(0.11,0.15)$ & 4.72 \\
\hline Yan Zhongrui 1998 & $\rightarrow$ & $0.08(0.06,0.10)$ & 4.73 \\
\hline Wang Liping 2005 & $\rightarrow$ & $0.11(0.09,0.13)$ & 4.75 \\
\hline Duan Kangli 2018 & $-\quad i$ & $0.05(0.04,0.06)$ & 4.88 \\
\hline Yang Junping 2014 & -1 & $0.10(0.08,0.12)$ & 4.75 \\
\hline Zhang Ming 2016 & $-\infty$ & $0.18(0.14,0.23)$ & 4.25 \\
\hline Wang Xiaoqing 2018 & in & $0.17(0.13,0.20)$ & 4.51 \\
\hline Chen Jingfei 233 & & $0.18(0.13,0.23)$ & 4.15 \\
\hline Xu Lina 2018 & & $0.14(0.11,0.18)$ & 4.41 \\
\hline Sun Shuju 2013 & 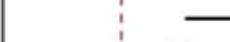 & $0.28(0.21,0.35)$ & 3.55 \\
\hline Xu Gelin 2007 & $\rightarrow-$ & $0.21(0.18,0.23)$ & 4.65 \\
\hline Wang Weiying 2016 & $\rightarrow$ & $0.11(0.08,0.14)$ & 4.58 \\
\hline Overall $(1$-squared $=97.9 \%, p=0.000)$ & & $0.14(0.11,0.16)$ & 100.00 \\
\hline
\end{tabular}

Figure 4 Cumulative risk of stroke recurrence 1 year after first-ever AIS

\section{Sensitivity analysis}

The cumulative recurrence rate at 3 months, 6 months, 1 year, 2 years, and 5 years after AIS were calculated respectively by the fixed effect model and the random effects model, as shown in Table 3. The results showed that the combined results of different effect models at 6 months, 5 years after AIS were consistent, suggesting that the results of meta analysis of cumulative recurrence rates were more stable and reliable. In the sensitivity analysis of cumulative recurrence rate at 3 months, 1 year and 2 years after AIS, it was found that the results of the meta-analysis of the 2 cumulative relapse rates were 


\begin{tabular}{|c|c|c|c|}
\hline $\begin{array}{l}\text { Study } \\
\text { ID }\end{array}$ & & ES $(95 \% \mathrm{Cl})$ & $\begin{array}{l}\% \\
\text { Weight }\end{array}$ \\
\hline $\begin{array}{l}\text { Zheng Jianghuan } 2015 \\
\text { Li Qi 2014 } \\
\text { Su Changqing } 2014 \\
\text { An Yachen } 2017 \\
\text { Chen Yunxia } 2011 \\
\text { Zheng Shengbang } 2017 \\
\text { Tang Meilian } 2016 \\
\text { Wang Liping } 2005 \\
\text { Yang Junping } 2014 \\
\text { Xue Min } 2013 \\
\text { Zhang Youlin } 2012 \\
\text { Overall (I-squared }=97.8 \%, p=0.000 \text { ) }\end{array}$ & + & $\begin{array}{l}0.19(0.13,0.25) \\
0.19(0.12,0.25) \\
0.25(0.18,0.33) \\
0.15(0.13,0.17) \\
0.19(0.16,0.23) \\
-\quad 0.30(0.27,0.34) \\
0.05(0.05,0.06) \\
0.16(0.14,0.19) \\
0.12(0.10,0.15) \\
0.15(0.12,0.18) \\
0.19(0.13,0.24) \\
0.17(0.12,0.23)\end{array}$ & $\begin{array}{l}8.57 \\
8.43 \\
7.97 \\
9.56 \\
9.22 \\
9.29 \\
9.71 \\
9.50 \\
9.53 \\
9.45 \\
8.77 \\
100.00\end{array}$ \\
\hline
\end{tabular}

Figure 5 Cumulative risk of stroke recurrence 2 years after first-ever AIS

\begin{tabular}{|c|c|c|c|}
\hline \multicolumn{3}{|l|}{ Study } & \multirow{2}{*}{$\begin{array}{l}\% \\
\text { Weight }\end{array}$} \\
\hline ID & & $\mathrm{ES}(95 \% \mathrm{Cl})$ & \\
\hline Cheng Yuefeng 2017 & & $\rightarrow 0.46(0.41,0.50)$ & 24.58 \\
\hline Zheng Shengbang 2017 & & $\rightarrow \quad 0.36(0.32,0.39)$ & 24.99 \\
\hline Run Zhongrui 1998 & $\rightarrow-$ & $0.20(0.16,0.23)$ & 25.15 \\
\hline Wang Liping 2005 & $\rightarrow$ & $0.23(0.20,0.26)$ & 25.28 \\
\hline Overall $(I-$ squared $=97.3 \%, p=0.000)$ & & $0.31(0.20,0.42)$ & 100.00 \\
\hline
\end{tabular}

Figure 6 Cumulative risk of stroke recurrence 5 years after first-ever AIS

inconsistent, suggesting that the stability coefficient of the results of meta analysis of the 2 cumulative recurrence rates were lower.

\section{Publication bias analysis}

The results of Egger's test showed that there might be publication bias in the literature which reported the cumulative recurrence rate at 6 months, 1 year and 2 years after the initial ischemic stroke $(P<0.05)$. However, there was no significant publication bias in the literature which reported the cumulative recurrence rate at 3 months and 5 years after the initial AIS $(P>0.05)$.

\section{Discussion}

Acute ischemic stroke has a higher recurrence rate. Compared with initial AIS patients, the mortality and disability rate of recurrent ischemic stroke patients are significantly increased, causing great troubles and burdens for patients and families. There are different reports on the recurrence rate of ischemic stroke in China, and the results of different literature reports are quite different. Therefore, it is necessary to summarize and analyze the recurrence rate of AIS in China. The study included 29 studies covering 19 provinces (cities and autonomous regions) in China, with a total of 22484 cases. The prevalence of recurrent ischemic stroke in China in recent years was well described in this study. Through meta analysis, the pooled cumulative recurrence risk at 3 months, 6 months, 1 year, 2 years, and 5 years after initial AIS in China was $4.5 \%, 7.8 \%, 13.6 \%, 17.5 \%$, and $30.9 \%$, respectively. According to the 2017 Chinese Stroke Prevention Report, the 1-year recurrence rate of patients with AIS in China was $13.2 \%^{[32]}$. The result is basically consistent with the result of this study, which also verified the accuracy and reliability of this study. However, a foreign meta analysis involving 13 studies $^{[1]}$ indicates that the pooled cumulative risk was $11.1 \%(95 \% \mathrm{CI}: 9.0-13.3)$ at 1 year, $26.4 \%$ (95\% CI: 20.1-32.8) at 5 years after initial stroke, which was lower than the pooled cumulative recurrence risk obtained in this study. It is speculated that the reasons for this difference may be related to the regional and ethnic differences in the recurrence of ischemic stroke and the different inclusion criteria of the 2 studies. Differences in sample sizes are also taken into account.

The study strictly follows the reporting specification of observational meta-analysis, but there are still some limitations that need to be explained. (1) Restricted to the characteristics of single-rate meta analysis, there may be a high degree of heterogeneity ${ }^{[33]}$. It is consistent with the meta analysis of single rate published at home and 
Table 3 Sensitivity analysis results

\begin{tabular}{ccccc}
\hline $\begin{array}{c}\text { Cumulative risk of stroke } \\
\text { recurrence }\end{array}$ & \multicolumn{2}{c}{ Fixed effect model } & \multicolumn{2}{c}{ Random effect model } \\
\cline { 2 - 5 } & Combined effect value & $\mathbf{9 5 \% ~ C I ~}$ & Combined effect value & 95\% CI \\
\hline 3 months after first-ever AIS & 1.036 & $1.031-1.042$ & 0.045 & $0.031-0.058$ \\
6 months after first-ever AIS & 0.054 & $0.048-0.061$ & 0.078 & $0.056-0.100$ \\
1 years after first-ever AIS & 0.049 & $0.046-0.052$ & 0.136 & $0.110-0.162$ \\
2 years after first-ever AIS & 0.079 & $0.074-0.083$ & 0.175 & $0.124-0.226$ \\
5 years after first-ever AIS & 0.280 & $0.263-0.297$ & 0.309 & $0.202-0.417$ \\
\hline
\end{tabular}

abroad ${ }^{[34-35]}$. (2) The results of the study were scattered. Considering that the cumulative recurrence rate of AIS was influenced by time factors, the total recurrence rate was not calculated in this study. And the subgroup analysis of sex, age, region and so on could not be carried out without calculating the total recurrence rate. (3) The severity of the patient's condition is different, resulting in a large heterogeneity among the studies. (4) Due to the fact that the literature included in this study were limited to publicly published and accepted research in the database, and some of the literature had shorter follow-up time, non-reported response rate, non-reported data integrity and methods of handling missing values, and so on, the publication bias of the literature relatively large. It may affect the accuracy of the results. Therefore, the results of this study still need to be further verified by random sampling and large-scale national epidemiological surveys.

To sum up, the recurrence risk after acute ischemic stroke in China is relatively high. It is suggested that medical and health departments at all levels must pay more attention to the prevention and treatment of recurrent ischemic stroke, and attach importance to the recurrence factors of ischemic stroke. We should take appropriate interventions to reduce the recurrence of ischemic stroke, improve the prognosis, lower the fatality rate and disability rate.

\section{Declaration}

The authors of this article declare no conflict of interest.

\section{References}

1 Mohan KM, Wolfe CDA, Rudd AG, et al. Risk and
Cumulative Risk of Stroke Recurrence A Systematic Review and Meta-Analysis. Stroke, 2011, 42(5): 1489-1494.

2 Wells G, Shea B, Connell D, et al. The NewcastleOttawa Scale (NOS) for Assessing the Quality of Non-randomised Studies in Meta-analyses. (Feb. 262018) [Online] Available from: http://www. ohri.ca/programs/clinical_epidemiology/oxford. htm[Accessed on March 182018].

3 Chen P. Analysis of risk factors for recurrence in 401 patients with ischemic stroke. Yinchuan: Ningxia Medical University, 2014.

4 Zheng JH. Risk factors for the recurrence of acute ischemic stroke and the predictive value of LDL/ HDL. Chin J Gerontol, 2015, (21): 6123-6124.

5 Li Q, Zhong CZ. Analysis of risk factors for the recurrence of acute ischemic stroke and the predictive value of the ratio of low-density lipoprotein and highdensity lipoprotein for the stroke recurrence. Chin J Circ, 2014, (9): 694 - 697.

6 Luan M, Wen ZC. Analysis of risk factors for the recurrence of patients with acute ischemic stroke and the construction of prognostic index model. J Clin Emerg, 2016, 17(8): 610-613.

7 Su CQ. Predictive factors for the recurrence of cerebral infarction. Fuzhou: Fujian Medical University, 2014.

8 Song L. Risk factors for recurrence of cerebral infarction. Dalian: Dalian Medical University, 2008.

9 Cheng YF, Lin L, Dong H, et al. Analysis of factors influencing recurrence in patients with cerebral infarction. Med Rev, 2017, 23(21): 4352-4355.

10 An YC, Cheng Y, Wang YX, et al. The Cox regression analysis of the risk factors for the 3-year recurrence of 
ischemic stroke and the construction of the prediction model. Chin J Behav Med Brain Sci, 2017, 26(6): 544-548.

11 Chen YX. Analysis of risk factors for the recurrence of is chemic stroke. Tangshan: Hebei Union University, 2011.

12 Zhang SN, Xu GL, Fan XY, et al. Analysis of risk factors for ischemic stroke. J Med Postgrad, 2008, 21(9): 962-965.

13 Zheng SB, Yan YJ, Li J, et al. Risk factors for the recurrence of cerebral infarction in Minhang District of Shanghai. Chin J Chronic Dis Prev Control, 2017, 25(8): 595-598.

14 Wang J. Analysis of recurrence and high risk factors in 1 year of first-episode ischemic stroke. Suzhou: Suzhou University, 2016.

15 Tang ML. Distribution characteristics of risk factors in inpatients with stroke in China, cumulative recurrence rate in the first four years and its influencing factors. Beijing: Capital Medical University, 2016.

16 Zhang CQ, Wang YL, Wang CX, et al. Analysis of risk factors for recurrence of non-cardiogenic ischemic stroke. Chin J Stroke, 2018, 13(1): 23-28.

17 Zhao Y, Su J, Fu ZX, et al. Analysis of risk factors in patients with recurrent cerebral infarction. Chinese Journal of practical Neuropathy, 2014, 17(17): 73-74.

18 Ma JJ, Tan YC, Geng DQ. Analysis of risk factors for short-term recurrence of ischemic stroke. Med Inform, 2015, (41): 17-18.

19 Yan ZR, Liu CY, Zhao L, et al. Study on the recurrence and risk factors of ischemic cerebrovascular disease. Stroke Neurop, 1998, (4): 192-195.

20 Wang LP, Zhu HM. Analysis of related factors of recurrent cerebral infarction. J Pract Diag Ther, 2005, (1): 58-59.

21 Duan KL, Liu ZZ, Lin XM, et al. Analysis of risk factors associated with 1-year stroke recurrence in patients with acute ischemic stroke in Xi'an area. $\mathrm{J}$ Shanxi Med Univ, 2018, 49(05): 539-543.

22 Yang JP, He GS. Discussion on the factors related to the recurrence of ischemic stroke. Public Medical Forum, 2014, 18(19): 2582-2583.

23 Zhang M. Factors of secondary prevention and recurrence in 196 patients with ischemic stroke. Jinan: Shandong University, 2016.

24 Xue M, Zhang M, Zhu L, et al. Related risk factors for the recurrence of ischemic stroke. Chin Basic Med, 2013, 20(14): 2081-2083.

25 Wang XQ. Analysis of the risk factors of recurrent stroke in 450 patients with ischemic stroke. Shijiazhuang: Hebei Medical University, 2016.

26 Chen JF, Chen GS. Recurrence and risk factors in patients with first-episode ischemic stroke. J Ningxia Med Univ, 2015, 37(5): 546-548.

$27 \mathrm{Xu}$ LN, Jia LB, Pang SX, et al. Study on related factors of the recurrence in patients with first-episode ischemic stroke. Chin J Pract Med, 2018, 45(15): 1113.

28 Zhang YL, Zheng H, Zeng YJ, et al. Risk factors for the recurrence in middle-aged patients with acute cerebral infarction. Shandong Med, 2012, 52(29): 6263.

29 Sun SJ, Zhou JL. Relationship between the characteristics of carotid artery disease and the recurrence in one year. Chin J Gerontol, 2013, 33(19): 4857-4858.

$30 \mathrm{Xu}$ G, Liu X, Wu W, et al. Recurrence after Ischemic Stroke in Chinese Patients: Impact of Uncontrolled Modifiable Risk Factors. Cerebrovasc Dis, 2007, 23(23): 117-120.

31 Wang WY, San WW, Yan SM, et al. Cox regression analysis of 1-year recurrence risk factors in patients with acute ischemic stroke. Chin J Cardiovasc Cerebrovasc Dis, 2016, 18(1): 46-50.

32 Wang LD, Liu JM, Yang Q, et al. Summary of 2017 report on prevention and treatment of stroke in China. Chin J Cerebrovasc Dis, 2018, 15(11): 611-617.

33 Shi XQ, Wang ZZ. Application of Meta-regression and subgroup analysis in heterogeneity processing. Chin J Epidemiol, 2008, 29(5): 497-501.

34 Ahmed B, Alkhaffaf H.Prevalence of significant asymptomatic carotid artery disease in patients with peripheral vascular disease: a metaanalysis. Eur J Vasc Endovasc Surg, 2009, 37(3): 262-71.

35 Han JM, Wang ZY, Yang L, et al. Meta analysis of depression rate in elderly population from Chinese hospital. Chin J Gerontol, 2019, 39(05): 1117-1121. 